\title{
Overexpression of SEZ6L2 predicts poor prognosis in patients with cholangiocarcinoma
}

\author{
Ziming Wang ${ }^{1}$, Jiaming Lai ${ }^{1}$, Lijian Liang ${ }^{1}$, Xiaoyu Yin ${ }^{1}$, Qian Wang ${ }^{1}$, Quanyong Cheng ${ }^{2}$, Chaoxu Zheng ${ }^{1}$ \\ ${ }^{1}$ Department of Pancreato-Biliary Surgery, The First Affiliated Hospital of Sun Yat-Sen University, Guangzhou, China; ${ }^{2}$ Center for Private Medical \\ Service \& Healthcare, The First affiliated Hospital of Sun Yat-sen University, Guangzhou, China \\ Contributions: (I) Conception and design: Q Cheng, C Zheng; (II) Administrative support: C Zheng; (III) Provision of study materials or patients: J \\ Lai, L Liang; (IV) Collection and assembly of data: Z Wang, Q Wang, Q Cheng; (V) Data analysis and interpretation: Z Wang, L Liang, C Zheng; (VI) \\ Manuscript writing: All authors; (VII) Final approval of manuscript: All authors. \\ Correspondence to: Prof. Chaoxu Zheng, MD \& PhD. The First Affiliated Hospital of Sun Yat-Sen University, Guangzhou, China. \\ Email: zhengcx@mail.sysu.edu.cn; Dr. Quanyong Cheng, MD. Center for Private Medical Service \& Healthcare, The First affiliated Hospital, Sun \\ Yat-Sen University, Guangzhou 510080, China. Email: yongshu88@sina.com.
}

Background: With the feature of destructive and biliary malignancy, intrahepatic cholangiocarcinoma (ICC), presents unclear molecular mechanisms which contributes to typically poor prognosis for patients. Seizure-related 6 bomolog-like 2 (SEZ6L2) is a gene that encodes for a seizure-associated protein localized on the cell surface. Thus far, the function of SEZ6L2 in ICC has not been reported.

Methods: We used data from The Cancer Genome Atlas and the Gene Expression Omnibus to analyze dynamics behind and levels of expression of SEZ6L2 in ICC. Then we used qRT-PCR and Immunohistochemical staining to detect levels of expression of SEZ6L2 and thereby determined the potential clinical significance of this protein in ICC.

Results: According to qRT-PCR and immunohistochemical analysis results, SEZ6L2 was overexpressed in ICC. Kaplan-Meier and Cox proportional hazard analyses indicated that patients afflicted by ICC with high levels of relative expression of SEZ6L2 have a poorer prognosis and that SEZ6L2 may be an independent prognostic factor which enables to the accurate prediction of overall survival (OS) and disease-free survivals' (DFS) expected rates. Subcutaneous xenograft models used to explore the role of SEZ6L2 in tumor formation in vivo. The dynamics of the SEZ6L2 gene being promote angiogenesis in cholangiocarcinoma are related to increasing expressive growth factors which include $E G F, V E G F, P D G F$ and the activation of the P38-MAPK pathway.

Conclusions: Our findings suggest that SEZ6L2 can serve as an advanced biomarker that can be used to accurately predict a patient prognosis and be used as a target for ICC treatment.

Keywords: Intrahepatic cholangiocarcinoma (ICC); seizure-related 6 bomolog-like 2 (SEZ6L2); prognostic factor; P38-MAPK pathway

Submitted Mar 16, 2020. Accepted for publication Sep 10, 2020.

doi: $10.21037 /$ tcr-20-1527

View this article at: http://dx.doi.org/10.21037/tcr-20-1527

\section{Introduction}

Intrahepatic cholangiocarcinoma (ICC) is an aggressive malignancy with poor prognosis for afflicted patients. The incidence of ICC has increased over recent years, ICC accounts for $15-20 \%$ of all primary hepatobiliary malignancies (1-3). According to some studies, several proteins, e.g., carbohydrate antigen (CA) 19-9, can serve as biomarkers that can be used in the process of accurate prognosis prediction $(4,5)$. Thus, further research to elucidate mechanisms related to the ICC progression is still 
needed, which can contribute to the development of new therapeutic strategies for afflicted patients. We have been screening for genes encoding up-regulated/down-regulated molecules in cholangiocarcinoma using microarrays derived from tumor cells purified by laser capture microdissection. To verify the clinicopathological and biological significance of the respective gene products, we performed tissue microarray analysis of clinical cholangiocarcinoma specises. This systematic approach combines bioinformatics tools to search for cell surface and/or secreted proteins and finds that SEZ6L2 is frequently transcribed in a large number of patients with cholangiocarcinoma.

Seizure-related 6 bomolog like 2 (SEZ6L2) is a gene that encodes a seizure-associated protein localized on the cell surface, which plays a role when it comes to the regulation of a variety of biological functions. SEZ6L2 is a type 1 membrane protein containing the $\mathrm{C} 1 \mathrm{r} / \mathrm{C} 1 \mathrm{~s}$, Uegf, and Bmp1 (CUB) complement domains as well as complement control protein (CCP) domains (6). Proteins which contain these domains help to regulate the localization or function of neurotransmitter receptors $(7,8)$, and serve as a helper subunit for $G l u R$ (9). Research indicated that the overexpression of $S E Z 6 L 2$ in lung cancer makes it a potential prognostic marker for this type of tumor (10), however the dynamics and function of SEZ6L2 in cholangiocarcinoma still remains unclear.

In our study, we sought to investigate the levels of expression of SEZ6L2 in ICC by assessing several ICC specimen types with paired para-cancerous tissues. The relationships between the level of expression of SEZ6L2 and clinical pathological features, overall survival (OS), and disease-free survival (DFS) of patients with ICC were also analyzed. We present the following article in accordance with the ARRIVE reporting checklist (available at http:// dx.doi.org/10.21037/tcr-20-1527).

\section{Methods}

\section{Patients and tissue samples}

We obtained all samples from patients with ICC at the First Affiliated Hospital of Sun Yat-Sen University (Guangzhou, China) between January, 2004 and December, 2015. All samples were from patients who underwent radical surgical resectioning without preoperative chemotherapy or radiotherapy. All patients were followed up with until at least through December 2017, and all initial and ongoing diagnoses were confirmed by using standard methodologies for pathological analysis. Complete clinical pathological and follow-up data for our study are available for all 101 ICC samples we collected. It conformed to the provisions of the Declaration of Helsinki (as revised in 2013). The study was approved by Institutional Research Ethics Committee of the First Affiliated Hospital of Sun Yat-sen University (2019-P220) and informed consent was taken from all the patients. All fresh tumor tissue specimens were rapidly frozen in liquid nitrogen immediately after resection and then stored at $-80^{\circ} \mathrm{C}$.

\section{Cell culture}

Human cholangiocarcinoma cell line RBE was purchased from the cell bank of Shanghai Academy of Chinese Sciences. The frozen cells were removed from liquid nitrogen before the experiment and thawed rapidly in a constant temperature water bath at $37^{\circ} \mathrm{C}$. During the experiment, the cells were shaken continuously to accelerate the thawing. Cell suspension was quickly transferred to a centrifugal tube containing $10 \mathrm{~mL}$ RPMI1640 medium containing $10 \%$ FBS in a super-clean workbench for mixing.

Cells were transferred to culture flask with a transpirator, and the algebra and date of cells were marked on the flask. The cells were placed in a $37^{\circ} \mathrm{C}, 5 \% \mathrm{CO} 2$ incubator for further culture.

\section{Quantitative real-time polymerase chain reaction (qRT-PCR)}

The fresh frozen tissue was lysed in TRIzol solution (Invitrogen, Carlsbad, CA, USA) to obtain purified RNA. $1 \mathrm{mg}$ of total RNA was transcribed reversely into cDNA with Verso cDNA Kit (Thermo Fisher Scientific). The relative gene expression level was monitored via qPCR by use of SYBR Premix Ex Taq II (TaKaRa, Japan) and Termal Cycler Dice TP850 (Takara) as per the standard methods provided by supplier. The $2-\Delta \Delta \mathrm{CT}$ method was applied for determination of target gene expression in experimental group and control group. qRT-PCR was conducted in triplicate for each sample, and detected gene expression was normalized to that of GAPDH.

\section{Plasmid, small interfering RNA and transfection}

The coding region of SEZ6L2 gene was cloned into 
PUC57 vector to construct the overexpression vector pBPLV-SEZ6L2. Three SEZ6L2 interfering plasmids were constructed, and the sequence of interfering plasmids was as follows.

shRNA1, CCGGCCAGCCCAGTTCTGTGTAATAC TCGAGTATTACACAGAACTGGGCTGGTTTTTG; shRNA2, CCGGGCTCCAAGTTGAGATATTGAAC TCGAGTTCAATATCTCAACTTGGAGCTTTTTG; shRNA3, CCGGCGTGATCTATGATTCGGACATCTC GAGATGTCCGAATCATAGATCACGTTTTTG. Then the interfering vector was transfected into RBE cells. After 96 hours, RNA was extracted from RBE cells for qPCR verification, and a plasmid with the highest efficiency was screened for subsequent experiments.

\section{Western blot analysis}

Western blot was used to detect the expression of SEZ6L2 protein and the expression of angiogenesis related factors CD 36, EGF, P38, PDGF, VEGF and VEGFR-1 after gene interference. The cells were collected and centrifuged, and the corresponding lysate was added. The cells were lysed at $4{ }^{\circ} \mathrm{C}$ for $30 \mathrm{~min}$ and centrifuged at 10,000 rpm for $10 \mathrm{~min}$. The supernatant was carefully absorbed to obtain the total protein. Protein concentration was determined by BCA kit. After boiling, sampling and electrophoresis for 1 to 2 hours, the protein was transferred to PVDF membrane. The membrane was incubated overnight with a solution of the first antibody at $4{ }^{\circ} \mathrm{C}$, and then the second antibody was incubated at room temperature for 1 to 2 hours. ECL exposure solution was dripped onto the membrane and exposed. Quantity one software was used to analyze the gray value of each antibody band.

\section{Immunobistochemical staining}

The de-paraffin-rehydrated sections were heated in sodium citrate buffer ( $\mathrm{pH}$ 6.0) for $10 \mathrm{~min}$ for antigen retrieval. After inactivation of endogenous peroxidase by $3 \%$ hydrogen peroxide, the antibody against SZE6L2 was incubated for $1 \mathrm{~h}$ at $37^{\circ} \mathrm{C}$. An IHC kit (Dako) was used to analyze the protein expression level as standard manual. Two more experienced pathologists scored the staining results. In this study, we subjected a total of 101 pairs of formalin-fixed paraffin-embedded ICC tissues to immunohistochemical staining.

\section{Animal studies}

Human cholangiocarcinoma cell line RBE $(0.2 \mathrm{~mL}$ PBS containing $5 \times 10^{5}$ cells) transfected with indicated plasmids was subcutaneously inoculated into the back of 5 -week-old female Balb/c nude mice (8 mice in each group). Tumor diameter was measured every 5 days in 20 days. The tumor volume $\left(\mathrm{mm}^{3}\right)$ was calculated based on the longest and shortest diameter. The formula was as follows: volume = (shortest diameter) $2 \times$ (longest diameter) $\times 0.5$. After 30 days of inoculation, the mice were killed and the xenografts were dissected for further analysis. Approval for the animal experiments was obtained from Sun Yat-sen University Animal Care and Use Committee, Institute of Clinical and Translational Research, Sun Yat-sen University (2019-P220). And the study was conducted in compliance with guidelines for the care and use of Laboratory Animals (Ministry of Health, People's Republic of China, 1998).

\section{Bioinformatic analysis}

Clinical information and data for levels of expression related to ICC were obtained from The Cancer Genome Atlas (TCGA; http://gdc.cancer.gov/) and from the Gene Expression Omnibus (GEO). We used R and GraphPad Prism 7 software to apply a log-transformation to the data and analyze the results. Negative binomial distributions, empirically based Bayesian estimations, exact tests, generalized linear models (GLMs) and quasi-likelihood tests in the edge $\mathrm{R}$ and limma packages were also used in this study to assess important patterns in the data. We used the metric of a calculated $\log \mathrm{FC}$ (fold-change) $\geq 1.0$ or $\log$ $\mathrm{FC} \leq-1.0$, and we defined an associated $\mathrm{P}$ value of $<0.05$ as a statistically significant gene.

\section{Statistical analysis}

We used SPSS version 23.0 (IBM) for statistical analysis. We used a Student's $t$-test to compare differences between treatment groups and explore the dynamics of the relationships between categorical variables with the $\chi^{2}$ test. The relative impact of various prognostic factors on the OS and DFS rates of patients with ICC was assessed using the Cox regression and Kaplan-Meier analysis. Two-tailed $\mathrm{P}$ values $<0.05$ were used to indicate statistically significant results at which the null hypothesis of no differences between treatment groups would be rejected. 

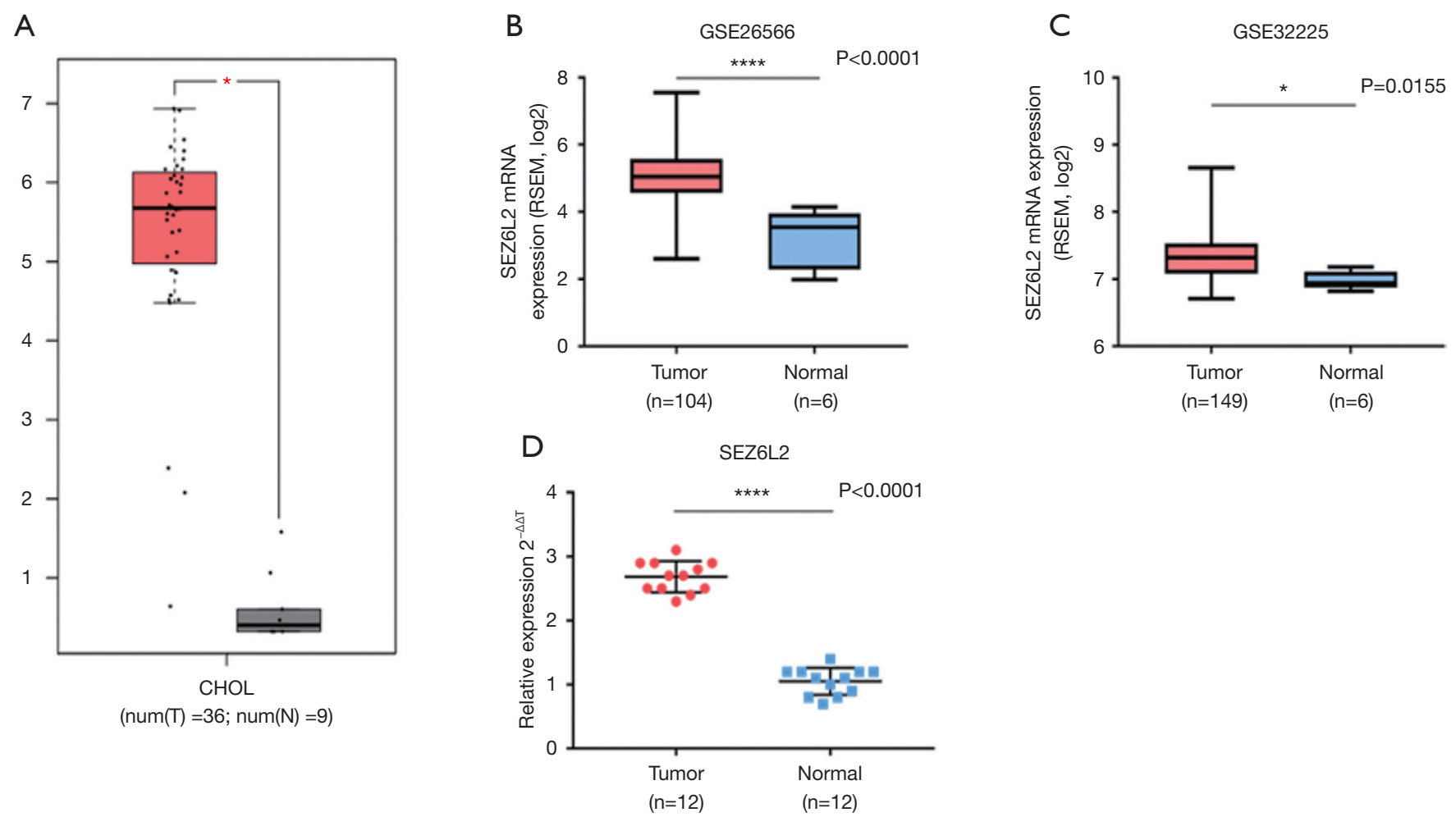

Figure 1 Upregulation of SEZ6L2 in intrahepatic cholangiocarcinoma. Analysis of data from The Cancer Genome Atlas indicates that SEZ6L2 is highly expressed in cholangiocarcinoma tissues compared with normal liver tissues $\left({ }^{*}, \mathrm{P}<0.05\right)(\mathrm{A})$ and an analysis of data from the Gene Expression Omnibus database (B,C; GSE26566, ****, $\mathrm{P}<0.0001$; GSE32225, *, P=0.0155). (D) Real-time PCR analysis of SEZ6L2 expression in 12 pairs of cholangiocarcinoma tissues and corresponding para-cancerous tissues $\left.{ }^{* * * *}, \mathrm{P}<0.0001\right)$.

\section{Results}

\section{The overexpression of SEZ6L2 in comparison with para- cancerous tissues}

We examined SEZ6L2 expression using mRNA sequencing or microarray datasets from TCGA and GEO to investigate levels of expression of SEZ6L2 in patients with ICC. Based on the results from analysis of TCGA, we compared 36 samples of ICC afflicted tissues with 9 samples of normal tissues, and the comparative results indicated that levels of expression of SEZ6L2 were significantly higher in tumor afflicted tissues than were respective levels in para-cancerous tissues (Figure 1A). In addition, the results from analysis of GSE26566 $(\mathrm{P}<0.0001)$ and GSE32225 $(\mathrm{P}=0.0155)$ indicated that the level of expression of SEZ6L2 was significantly elevated in ICC afflicted tissue sample compared with paracancerous tissues (Figure 1B,C). Moreover, we used qRTPCR and 12 pairs of fresh clinical samples which verified upregulation of the levels of expression of SEZ6L2 in tumor tissues (Figure 1D). The results of immunostaining analyses reflect that levels of expression of SEZ6L2 were high in 51 of the 101 total ICC cases in our study (Figure 2A; Table 1). Taken together, these results indicated that SEZ6L2 is overexpressed in ICC afflicted tissues comparing with the respective levels of expression in paired samples of paracancerous tissues.

\section{Correlations between SEZ6L2 expression and clinical parameters in ICC}

We next analyzed the immunostaining results and corresponding clinical data for the 101 pairs of ICC afflicted and non-diseased tissues to assess relationships between the levels of expression of SEZ6L2 and standardized ICC clinicopathological parameters. These clinical features are presented in Table 1. Results indicated that the level of expression of SEZ6L2 was strongly positively related to tumor size $(\mathrm{P}=0.002)$, lymphatic metastasis $(\mathrm{P}=0.016)$ and 
A

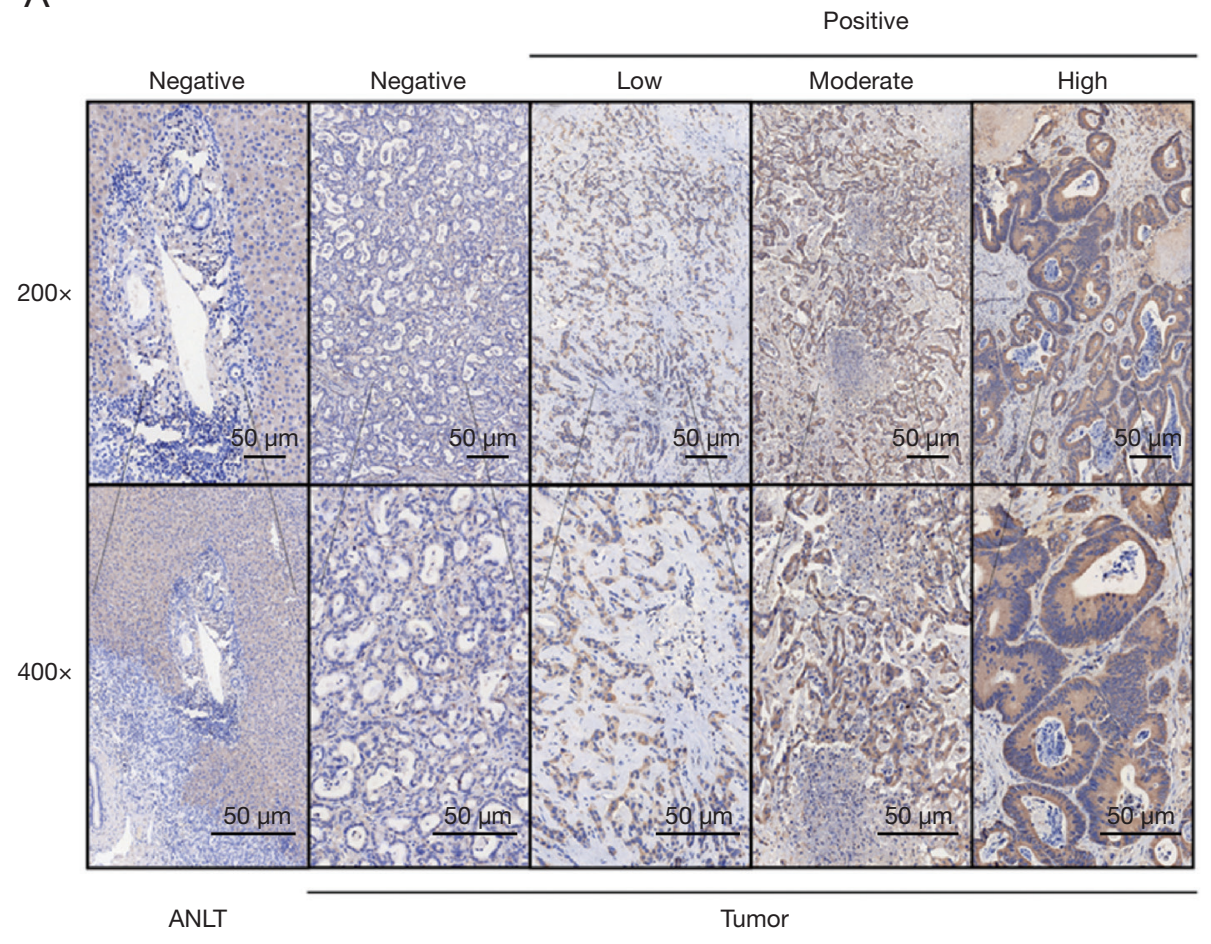

B

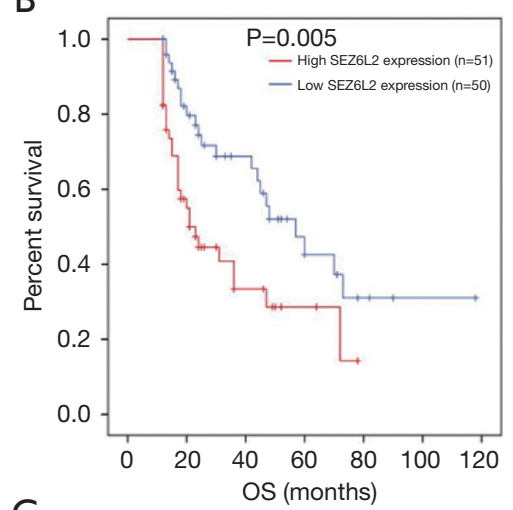

C



Figure 2 High SEZ6L2 expression predicts poor survival in patients with cholangiocarcinoma. Expression of SEZ6L2 in cholangiocarcinoma tissues and adjacent normal liver tissues (A). Overall survival and disease-free survival curves for ICC patient groups with low and high SEZ6L2 expression (B,C).

TNM stage $(\mathrm{P}=0.018)$. However, there we observed no significant difference with regard to the level of expression of SEZ6L2 and patient measures of sex, age, liver cirrhosis, or perineural and vascular invasion.

\section{SEZ6L2 is an independent prognostic marker in ICC patients}

To determine whether SEZ6L2 would be a good candidate to be used as an independent prognostic factor for treatment of patients with ICC, we utilized Cox regression analysis was utilized to examine OS and DFS. Univariate analysis indicated the stage of TNM, the levels of expression of SEZ6L2, and the degree of lymphatic metastasis was associated with OS. Furthermore, results from multivariate analysis indicated that the stage of TNM, medical complications, and levels of expression of SEZ6L2 were independent prognostic factors for OS (Table 2). As shown in Table 3, univariate analysis indicated that the levels of expression of SEZ6L2 and the stage of TNM are related to DFS. Multivariate analysis also indicated that the level of expression of SEZ6L2 was an independent prognostic factor for patients with ICC (Table 3). Taken together, the results of this aspect of our study indicate that SEZ6L2 can serve as an independent prognostic factor for patients with ICC.

\section{Poor survival prediction of strong SEZ6L2 expression in patients with ICC}

We next evaluated the prognostic value of the levels of expression of SEZ6L2 in patients with ICC using the Kaplan-Meier analysis. The data of 101 patients including survival data were sampled and analyzed, the results indicated that patients with a high level of expression of SEZ6L2 had correspondingly lower rates of OS and DFS than those with a low level of expression of SEZ6L2 ( $\mathrm{P}=0.005$ and $\mathrm{P}=0.024$ respectively; Figure $2 B, C)$. Thus, a high level 
Table 1 Correlation between SEZ6L2 expression and the clinicopathologic variables of 101 Intrahepatic cholangiocarcinoma patients

\begin{tabular}{|c|c|c|c|}
\hline \multirow{2}{*}{ Variables } & \multicolumn{2}{|c|}{ SEZ6L2 expression } & \multirow{2}{*}{$P$ value } \\
\hline & Low $(n=50)$ & High $(n=51)$ & \\
\hline \multicolumn{4}{|l|}{ Gender } \\
\hline Male & 20 & 29 & 0.090 \\
\hline Female & 30 & 22 & \\
\hline \multicolumn{4}{|c|}{ Age (years) } \\
\hline$<60$ & 26 & 27 & 0.925 \\
\hline$\geq 60$ & 24 & 24 & \\
\hline \multicolumn{4}{|c|}{ Lymphatic metastasis } \\
\hline Absent & 38 & 27 & $0.016^{*}$ \\
\hline Present & 12 & 24 & \\
\hline \multicolumn{4}{|c|}{ Tumor size $(\mathrm{cm})$} \\
\hline$\geq 2$ & 17 & 33 & $0.002^{*}$ \\
\hline$<2$ & 33 & 18 & \\
\hline \multicolumn{4}{|c|}{ Liver cirrhosis } \\
\hline Absent & 10 & 15 & 0.273 \\
\hline Present & 40 & 36 & \\
\hline \multicolumn{4}{|c|}{ Perineural and vascular invasion } \\
\hline Absent & 17 & 13 & 0.349 \\
\hline Present & 33 & 38 & \\
\hline \multicolumn{4}{|c|}{ TNM stages } \\
\hline$|\&| \mid$ & 36 & 25 & $0.018^{\star}$ \\
\hline III \& IV & 14 & 26 & \\
\hline Total & 50 & 51 & \\
\hline
\end{tabular}

*, significant difference.

of expression of SEZ6L2 appeared to successfully predict poor survival rates in patients with ICC.

\section{SEZ6L2 promoting ICC tumor growth in vivo}

The expression of GFP green fluorescent protein was the strongest when lentivirus SEZ6L2 was transfected into cholangiocarcinoma cell line RBE and assessed under inverted fluorescence microscopy. The efficiency of transfection of lentivirus SEZ6L2 knockdown vector was observed to $=75 \%$ (Figure $3 A$ ). Eighteen female nude mice aged 5-9 weeks were divided into an experimental group (transfected sh-SEZ6L2) and a negative control group (noload lentivirus, $\mathrm{N}=8$ mice each group). Results indicated that there were no significant differences in growth rates of transplanted tumors between the two treatment groups within 5 days after transplantations $(\mathrm{P}>0.05)$. After 10 days of transplantation, the growth rate of transplanted tumors in the experimental group was slower and smaller than those in the negative control group $(\mathrm{P}<0.05$, Figure $3 B, C, D)$. Immunohistochemical results showed positive expression of SEZ6L2 and vascular endothelial growth factor (VEGF) as observed through the appearance of obvious yellow and brown granules in the cytoplasm and as some cell membranes were stained (data not shown). Accordingly, the levels of expression of SEZ6L2 and CD34 in the experimental group were significantly lower than those respective levels in the negative control groups (Figure 3E,F).

\section{SEZ6L2 expression promoting angiogenesis of cholangiocarcinoma}

The results from qRT-PCR analysis indicated that the levels of expression of CD36, EGF, P38, PDGF, SEZ6L2, $V E G F$, and $V E G F R-1$ in the expression vector group were significantly higher than those respective levels in the control group and the empty vector treatment group $(\mathrm{P}<0.01$, Figure $4 A)$. In contrast, the effect of the interference vector group was opposite, and the levels of expression of related genes were inhibited successfully and found to be significantly different among the treatment groups $(\mathrm{P}<0.01$, Figure $4 B)$.

Results from western blotting showed that the levels of expression of CD36, EGF, P38, PDGF, SEZ6L2, VEGF, and $V E G F R-1$ in the expression vector group increased significantly comparing with the control and the empty vector groups $(\mathrm{P}<0.01$, Figure $4 C)$. The interference vector group had the opposite effect, which successfully inhibited expression of CD36, EGF, P38, PDGF, SEZ6L2, VEGF, and $V E G F-1$. The scientific significance $(\mathrm{P}<0.01$, Figure $4 D)$ was consistent with the results of qRT-PCR.

\section{Discussion}

Typically poor prognosis for patients with ICC is characterized with the feature of a median survival rate for patients who do not undergo surgery within 6 months, and characterized by a 5 -year survival rate for patients following complete resection of only $20 \%$ to $40 \%$ (11-13). According 
Table 2 Cox proportional-hazard regression analysis for overall survival

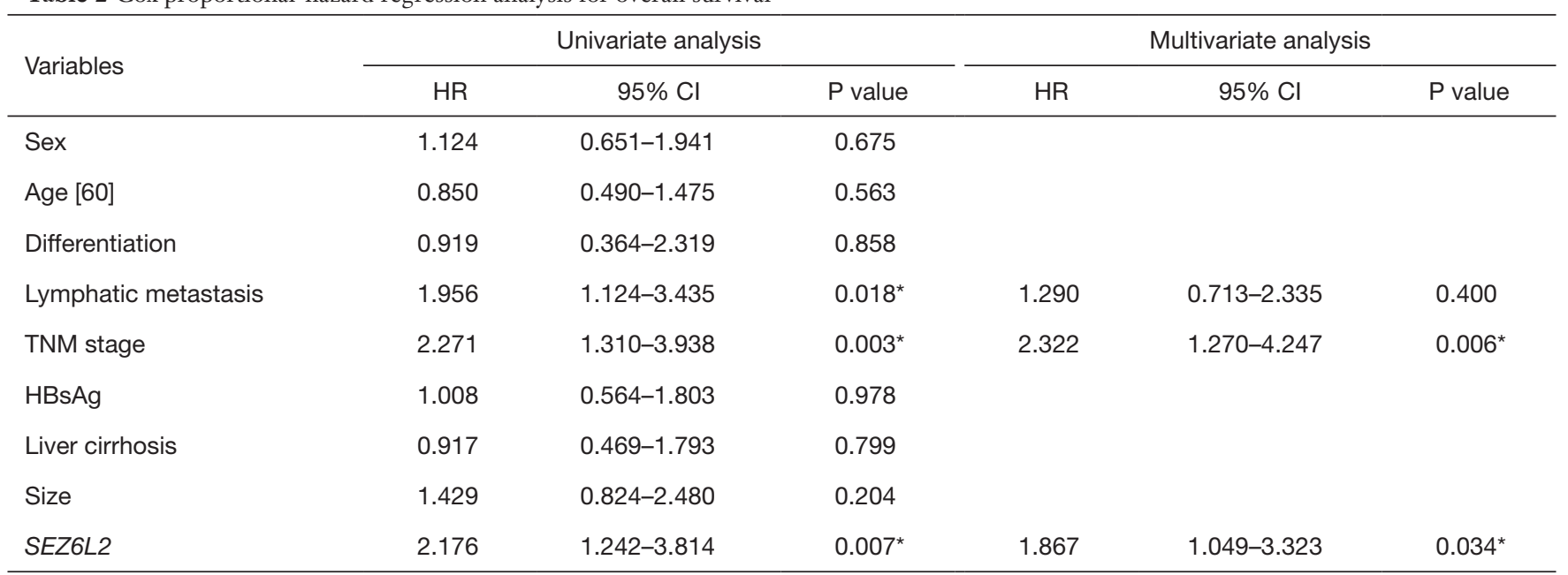

Lymphatic metastasis, TNM stage, SEZ6L2 are included in the Multivariate analysis. *, significant difference.

Table 3 Cox proportional-hazard regression analysis for disease-free survival

\begin{tabular}{|c|c|c|c|c|c|c|}
\hline Variables & \multicolumn{3}{|c|}{ Univariate analysis } & \multicolumn{3}{|c|}{ Multivariate analysis } \\
\hline Sex & 1.056 & $0.613-1.821$ & 0.843 & & & \\
\hline Age [60] & 0.765 & $0.440-1.330$ & 0.343 & & & \\
\hline Differentiation & 0.840 & $0.333-2.122$ & 0.713 & & & \\
\hline TNM stage & 1.988 & $1.151-3.438$ & $0.014^{\star}$ & 1.781 & $1.024-3.190$ & $0.042^{*}$ \\
\hline $\mathrm{HBsAg}$ & 0.855 & $0.478-1.530$ & 0.598 & & & \\
\hline Liver cirrhosis & 0.943 & $0.483-1.839$ & 0.862 & & & \\
\hline Size & 1.545 & $0.890-2.680$ & 0.122 & & & \\
\hline
\end{tabular}

Lymphatic metastasis, TNM stage, SEZ6L2 are included in the multivariate analysis. *, significant difference.

to previous research, the poor prognosis for patients with ICC is largely resultant due to the high frequency of recurrence and metastasis after surgical resection, as well as the resistance to systemic courses of chemotherapy-based treatments (14). Therefore, the identification of heretofore mostly unknown potential biomarkers that could be used for aiding early diagnosis of cases of ICC is urgently needed. By referring to the literature, it was reported that in non-small cell lung cancers (NSCLC), patients whose tumours revealed a higher level of SEZ6L2 expression suffered shorter tumour-specific survival compared to those with no SEZ6L2 expression (15). In addition, SEZ6L2 was significantly up-regulated in tumour tissues of patients with colorectal cancer compared with adjacent normal tissues. Up-regulation of SEZ6L2 was correlated with a poor prognosis in patients with colorectal cancer. Meanwhile, SEZ6L2 overexpression was closely associated with poor OS and DFS in hepatocellular carcinoma patients. Moreover, SEZ6L2 is an independent prognostic predictor for the survival of HCC patients (16). Until now, the clinical significance of SEZ6L2 in ICC remains uncertain and unclear. However, in our study, it is found that the levels of expression of SEZ6L2 were higher in ICC afflicted tissues comparing with the ANLTs samples. Taking all these 
A

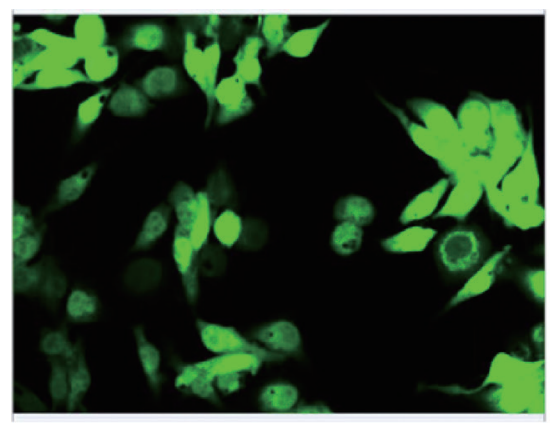

D

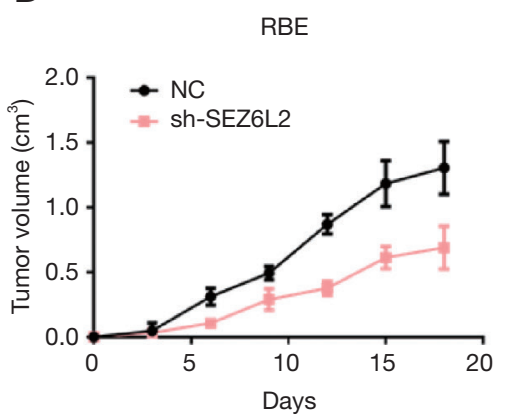

B

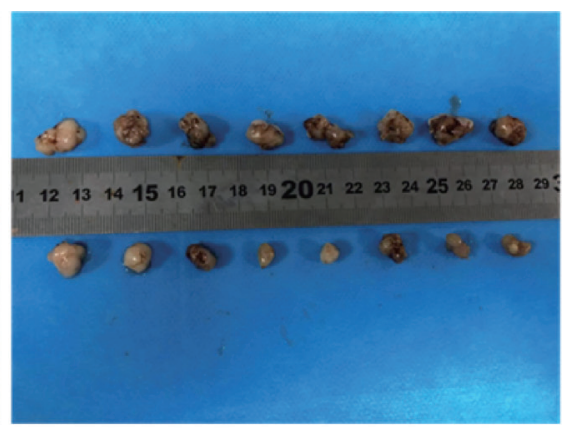

E

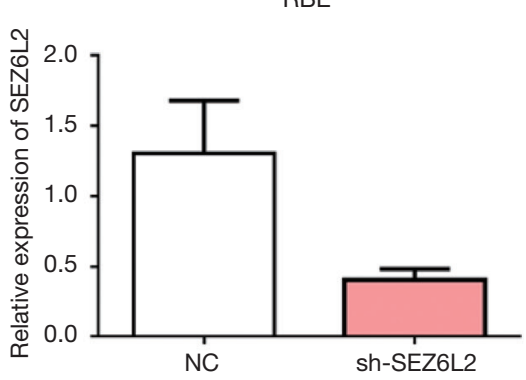

C



F

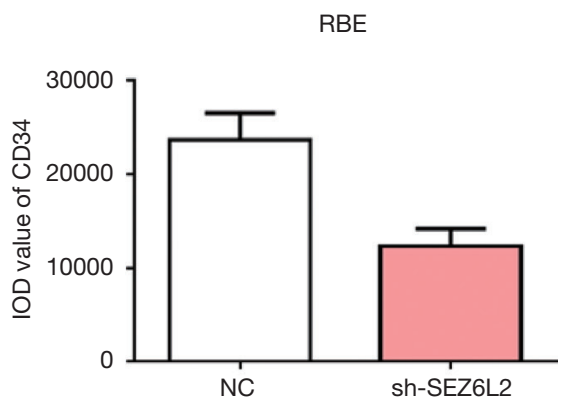

Figure 3 Knockdown of SEZ6L2 impaired cholangiocarcinoma carcinogenesis in vivo. The expression of GFP green fluorescent protein and assessed under inverted fluorescence microscopy after lentivirus was transfected into cholangiocarcinoma cell line RBE, the efficiency of transfection of lentivirus SEZ6L2 knockdown vector was observed to $=80 \%$ (A). The nude mice were sacrificed 28 days after the injection and tumors from respective groups were shown $(\mathrm{B}, \mathrm{C})$. Tumor growth curves after the injection of RBE cells. Tumor volume was calculated every 3 days (D). Relative expression was measured in IHC staining of SEZ6L2 in tumors with different treatments (E). Relative expression was measured in IHC staining of CD34 in tumors with different treatments.

results into consideration, survival data analysis indicated that SEZ6L2 over expression plays a significant role in the corresponding observation of poor survival rates of patients with ICC. These findings lead to the conclusion that SEZ6L2 is highly expressed in ICC afflicted tissues and can serve as a potential biomarker for promoting outcomes and accurate prognoses for patients with ICC.

$S E Z 6 L 2$ is a brain-specific receptor-like protein that is predominantly expressed in the soma and dendrites of neurons, which required for synaptic maturation (6). A previous study indicated that the promotion and regulation of SEZ6L2 methylation contributes to cell proliferation and metastasis through the regulation of dynamics in the pathways for STAT3, EGR1, and PAX4, and thus played an important role in the dynamics of pathogenesis of osteosarcoma (17). Both DNA methylation and histone modification are epigenetic modifications that can control gene expression and promote cell proliferation (18), cell differentiation, and cell adaptation to environmental conditions. Dysregulation of these processes may lead to various pathologies (19). So far, isoforms functions of SEZ6L2 have not been clarified in great detail. Therefore, further studies are still needed to confirm the results of our studies and hypotheses hypothesis related to the use of SEZ6l2 to improve the prognosis of patients with ICC.

SEZ6L2 encodes a $92.5-\mathrm{kD}$ a protein with an $\mathrm{N}$-terminal signal peptide, five SUSHI domains, three CUB domains, and a C-terminal transmembrane domain $(20,21)$. These encoded domains and products are likely mainly involved in developmental processes, cell to cell interactions, and dynamics related to cell adhesion $(22,23)$. It is known that cell to cell interaction and cell adhesion are strongly related to proliferation and metastasis, and may play additional potentially critical roles in tumor development and malignant transformations. In particular, the leading cause of death among cancer patients is metastasis (24). 
A

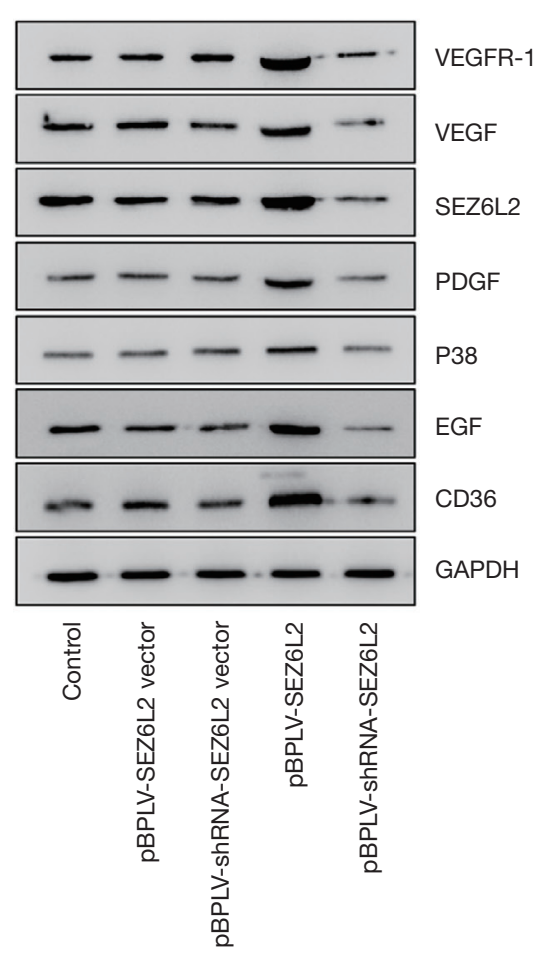

B
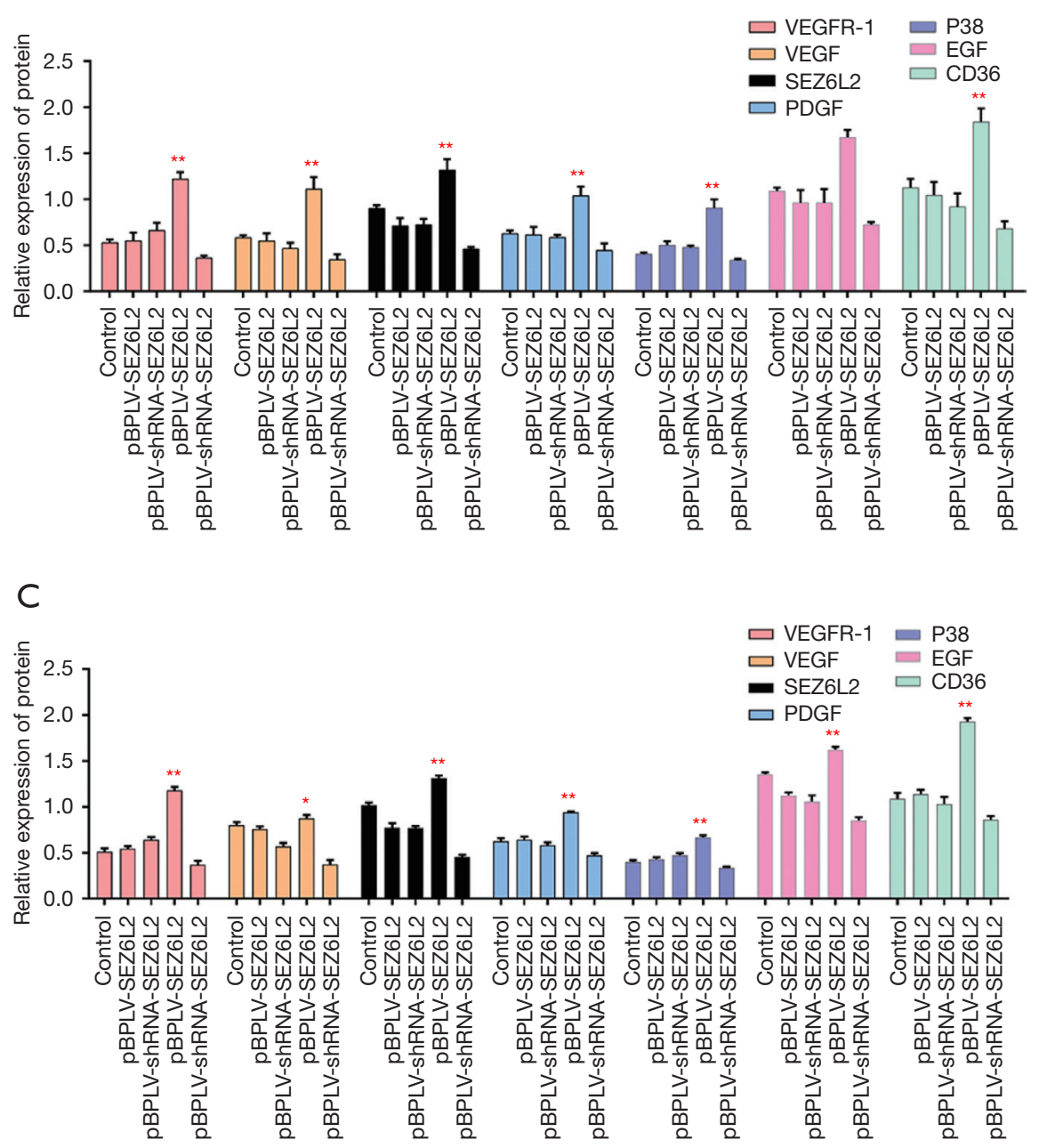

Figure 4 SEZ6L2 promoted cholangiocarcinoma progression by up-regulating angiogenesis. Western blot experiment shows that SEZ6L2 was positive correlated with VEGFR-1,VEGF, PDGF, p38, EGF and CD36. Knockdown of SEZ6L2 inhibited the protein expression of VEGFR-1, VEGF, PDGF, p38, EGF and CD36 (A)-(B). qPCR experiment shows that SEZ6L2 was positive correlated with VEGFR-1, VEGF, $P D G F, p 38, E G F$ and $C D 36$ and Knockdown of SEZ6L2 inhibited the mRNA expression of VEGFR-1,VEGF, PDGF, p38, EGF and CD36 (C). (All $\left.{ }^{*}, \mathrm{P}<0.05,{ }^{* *}, \mathrm{P}<0.01\right)$.

Additionally, cell migration and the ability of cancer cells to disseminate from the primary site forming distant metastases, are the primary causes of cancer-related morbidity in patients with solid tumors (25). Until now, the impact of SEZ6L2 on ICC and its mechanisms were unreported in the literature, and although our novel results are interesting, further investigations will still be required. However, this study clearly demonstrated that SEZ6L2 has potential for clinical use as a reliable biomarker for prognosis and investigations of ICC.

In our study, functional and immunohistochemical analysis of candidate molecules on tissue microarray has revealed that SEZ6L2 is likely to be a potential target for development of novel tools for diagnosis of ICC. In addition, in vitro and in vivo results suggested that SEZ6L2 functioned as an oncogene in cholangiocarcinoma patients. The knockdown of SEZ6L2 impairs tumour growth by promoting angiogenesis, which indicates that $S E Z 6 L 2$ could be a potential therapy target for ICC.

By designing small molecule inhibitors or gene therapy targeting SEZ6L2 (knockout of oncogene SEZ6L2), the therapeutic purpose of inhibiting tumorigenesis and 
development can be achieved. In addition, the application of tumor-targeting nanocarrier system to encapsulate chemotherapeutic drugs and gene-related agents (such as SEZ6L2 siRNA) for co-delivery of drug agents to tumor cell targets to achieve synergistic cancer suppression by chemotherapy and gene therapy is also a highly desirable approach for cancer treatment.

Cytokines including fibroblast growth factor-2, vascular endothelial growth factor (VEGF), EGF, and TGF-beta can regulate the levels of expression of SEZ6L2 (26,27). $V E G F$ is one of the strongest VEGFs. The combination of $V E G F$ and its receptor, $V E G F R$, specifically stimulates mitosis of vascular endothelial cells and promotes angiogenesis. Furthermore, as a powerful tumor lymph angiogenesis factor, $V E G F$ promotes proliferation of lymphatic system tumors, as well as induces changes in the levels of expression in tumor tissues and tumors. It has also been determined that there is a close relationship between the degree of vascularization and biological behaviors including the rates and degrees of infiltration and metastasis (28). PDGF is a receptor tyrosine kinase, which plays an important role in the growth, proliferation, and differentiation of cells including vascular endothelial cells by binding with receptors. $P D G F$ also promotes angiogenesis of tumors, thus promoting the growth, invasion, and metastasis of tumors.

Therefore, we used qPCR and western blot to detect the changes of the expression levels of EGF, P38, PDGF, SEZ6L2, VEGF, and VEGF receptor-in from determinations of protein and mRNA levels after SEZ6L2 interference and induced overexpression. The analytical results indicated that the levels of expression of VEGF, VEGFR, and PDGF were significantly increased after the SEZ6L2 gene was applied with induced interference and overexpression. In some cancer cells, there is a positive feedback mechanism of regulation between TGF-beta and SEZ6L2. These results imply that SEZ6L2 may promote the expression of $V E G F$, $V E G F$ receptor, and $P D G F$ through such a positive feedback regulation, thus ultimately promoting the angiogenesis of cholangiocarcinoma.

The results of our study lead to the conclusion that SEZ6L2 overexpression can be used as a metric to accurately predict a poor prognosis for patients with ICC. Furthermore, observations indicate that SEZ6l2 can serve as an independent prognostic factor for predicting outcomes related to OS and DFS in patients with ICC. Nonetheless, further work is needed to elucidate the molecular mechanisms through how SEZ6L2 promotes the progression of ICC.

\section{Acknowledgments}

The authors would like to thank Professor Wen Li (Laboratory of General Surgery, the First affiliated Hospital of Sun Yat-sen University, Guangzhou, China) for providing technical assistance.

Funding: None.

\section{Footnote}

Reporting Checklist: The authors have completed the ARRIVE reporting checklist. Available at http://dx.doi. org/10.21037/tcr-20-1527

Data Sharing Statement: Available at http://dx.doi. org/10.21037/tcr-20-1527

Conflicts of Interest: All authors have completed the ICMJE uniform disclosure form (available at http://dx.doi. org/10.21037/tcr-20-1527). The authors have no conflicts of interest to declare.

Ethical Statement: The authors are accountable for all aspects of the work in ensuring that questions related to the accuracy or integrity of any part of the work are appropriately investigated and resolved. The study was conducted in accordance with the Declaration of Helsinki (as revised in 2013). Approval and consent obtained for the use of human tissue were obtained from the Institutional Research Ethics Committee of the First Affiliated Hospital of Sun Yat-sen University (2019-P-220). Informed consent was taken from all the patients. Approval for the animal experiments was obtained from Sun Yat-sen University Animal Care and Use Committee, Institute of Clinical and Translational Research, Sun Yat-sen University (2019$\mathrm{P}-220)$. The study was conducted in compliance with guidelines for the care and use of Laboratory Animals (Ministry of Health, People's Republic of China, 1998).

Open Access Statement: This is an Open Access article distributed in accordance with the Creative Commons Attribution-NonCommercial-NoDerivs 4.0 International License (CC BY-NC-ND 4.0), which permits the noncommercial replication and distribution of the article with 
the strict proviso that no changes or edits are made and the original work is properly cited (including links to both the formal publication through the relevant DOI and the license). See: https://creativecommons.org/licenses/by-nc-nd/4.0/.

\section{References}

1. Tyson GL, El-Serag HB. Risk factors for cholangiocarcinoma. Hepatology 2011;54:173-84.

2. Everhart JE, Ruhl CE. Burden of digestive diseases in the United States Part III: Liver, biliary tract, and pancreas. Gastroenterology 2009;136:1134-44.

3. Rizvi S, Khan SA, Hallemeier CL, et al. Cholangiocarcinoma - evolving concepts and therapeutic strategies. Nat Rev Clin Oncol 2018;15:95-111.

4. Charatcharoenwitthaya P, Enders FB, Halling KC, et al. Utility of serum tumor markers, imaging, and biliary cytology for detecting cholangiocarcinoma in primary sclerosing cholangitis. Hepatology 2008;48:1106-17.

5. Brandi G, Venturi M, Pantaleo MA, et al. Cholangiocarcinoma: Current opinion on clinical practice diagnostic and therapeutic algorithms. Dig Liver Dis 2016;48:231-41.

6. Miyazaki T, Hashimoto K, Uda A, et al. Disturbance of cerebellar synaptic maturation in mutant mice lacking BSRPs, a novel brain-specific receptor-like protein family. Febs Lett 2006;580:4057-64.

7. Nakayama M, Hama C. Modulation of neurotransmitter receptors and synaptic differentiation by proteins containing complement-related domains. Neurosci Res 2011;69:87-92.

8. Nakayama M, Suzuki E, Tsunoda S, et al. The Matrix Proteins Hasp and Hig Exhibit Segregated Distribution within Synaptic Clefts and Play Distinct Roles in Synaptogenesis. J Neurosci 2016;36:590-606.

9. Zheng Y, Brockie PJ, Mellem JE, et al. SOL-1 is an auxiliary subunit that modulates the gating of GLR-1 glutamate receptors in Caenorhabditis elegans. Proc Natl Acad Sci U S A 2006;103:1100-5.

10. Ben-Menachem, Tamir. Risk factors for cholangiocarcinoma. Eur J Gastroenterol Hepatol 2007;19:615-7.

11. Yamamoto M, Ariizumi SI. Surgical outcomes of intrahepatic cholangiocarcinoma. Surg Today 2011;41:896-902.

12. Farges $\mathrm{O}$, Fuks D. Clinical presentation and management of intrahepatic cholangiocarcinoma. Gastroenterol Clin
Biol 2010;34:191-9.

13. Cardinale V, Semeraro R, Torrice A, et al. Intra-hepatic and extra-hepatic cholangiocarcinoma: New insight into epidemiology and risk factors. World J Gastrointest Oncol 2010;2:407-16.

14. Wang W, Fu L, Li S, et al. Histone deacetylase 11 suppresses p 53 expression in pituitary tumor cells. Cell Biol Int 2017;41:1290-5.

15. Ishikawa N, Daigo $\mathrm{Y}$, Takano A, et al. Characterization of SEZ6L2 cell-surface protein as a novel prognostic marker for lung cancer. Cancer Sci 2006;97:737-45.

16. Patil MA, Chua MS, Pan KH, et al. An integrated data analysis approach to characterize genes highly expressed in hepatocellular carcinoma. Oncogene 2005;24:3737-47.

17. Wang Q. CpG methylation patterns are associated with gene expression variation in osteosarcoma. Mol Med Rep 2017;16:901-7.

18. Nowacka-Zawisza M, Wisnik E. DNA methylation and histone modifications as epigenetic regulation in prostate cancer (Review). Oncol Rep 2017;38:2587-96.

19. Yanginlar C, Logie C. HDAC11 is a regulator of diverse immune functions. Biochim Biophys Acta Gene Regul Mech 2018;1861:54-9.

20. Clark HF, Gurney AL, Abaya E, et al. The secreted protein discovery initiative (SPDI), a large-scale effort to identify novel human secreted and transmembrane proteins: a bioinformatics assessment. Genome Res 2003;13:2265-70.

21. Norman DG, Barlow PN, Baron M, et al. Threedimensional structure of a complement control protein module in solution. J Mol Biol 1991;219:717-25.

22. Kirkitadze MD, Barlow PN. Structure and flexibility of the multiple domain proteins that regulate complement activation. Immunol Rev 2001;180:146-61.

23. Duke-Cohan JS, Gu J, McLaughlin DF, et al. Attractin (DPPT-L), a member of the CUB family of cell adhesion and guidance proteins, is secreted by activated human $T$ lymphocytes and modulates immune cell interactions. Proc Natl Acad Sci U S A 1998;95:11336-41.

24. Li R, Peng C, Zhang X, et al. Roles of Arf6 in cancer cell invasion, metastasis and proliferation. Life Sci 2017;182:80-4.

25. Jacquemet G, Hamidi H, Ivaska J. Filopodia in cell adhesion, 3D migration and cancer cell invasion. Curr Opin Cell Biol 2015;36:23-31.

26. Oh EK, Kim YW, Kim IW, et al. Differential DNA copy number aberrations in the progression of cervical lesions to invasive cervical carcinoma. Int J Oncol 2012;41:2038-46. 
27. Serizawa M, Takahashi T, Yamamoto N, et al. Genomic aberrations associated with erlotinib resistance in non-small cell lung cancer cells. Anticancer Res 2013;33:5223-33.

Cite this article as: Wang Z, Lai J, Liang L, Yin X, Wang Q, Cheng Q, Zheng C. Overexpression of SEZ6L2 predicts poor prognosis in patients with cholangiocarcinoma. Transl Cancer Res 2020;9(11):6768-6779. doi: 10.21037/tcr-20-1527
28. Skobe M, Hawighorst T, Jackson DG, et al. Induction of tumor lymphangiogenesis by VEGF-C promotes breast cancer metastasis. Nat Med 2001;7:192-8. 